\title{
Reducing Cognitive Load in Virtual Learning Environment
}

\author{
Jianzhen Chen \\ Tianjin University of Technology and Education, Tianjin, China \\ Xiaoping Li \\ Beijing Institute of Technology, Beijing, China
}

\begin{abstract}
Virtual reality provides a metaphorical parallel to our real world. The learning of source knowledge can be made easier by its metaphorical embodiment in artificial worlds. Our cognitive is the availability of a large store of organized information with practically unlimited capacity and duration. There are four factors of influencing cognitive load in virtual learning environment: learning tools, learning agents, learning activity and resource architecture .Learning tools are critical to manipulating the needed resources. Utilizing an agent based learner location process can help individual learner process new information. Learning activity as scaffolding has been used to facilitate learners in coping with complex problems. Resource architecture is to help people find and manage information more successfully.
\end{abstract}

KEYWORD: Cognitive Load; Virtual Learning Environment (VLE); Metaphor; Resource Architecture

\section{INTRODUCTION}

There has been a spectacular growth in the development of virtual reality environment for education over recent years. Ever since it appeared on the scene, the aim of virtual reality technology has been to build synthetic worlds capable of simulating, representing or recreating the different faces and sides of reality.

Source knowledge represents all the concepts, didactic materials, skills and information related to the subject to be learnt by the student. The real environment can be defined as the setting in which teaching takes place. This setting must be taken into account when selecting and studying the source knowledge. The educational environment can sometimes play a fundamental role and determine the forms of learning. Visualization of knowledge in virtual learning environment (VLE) is concerned with exploring information in such a way as to gain an understanding and insight into the data.

However, although virtual reality is a very versatile technology, it should not be used indiscriminately in any educational program. Human cognitive architecture is characterized by several major features that are essential for understanding cognitive processes in learning and performance. An essential characteristic of our cognitive architecture is the availability of a large store of organized information with practically unlimited capacity and duration. The concept of long-term memory (LTM) is traditionally associated with the storage of organized knowledge base in the form of hierarchical schematic knowledge structures[1].

Our cognitive system also includes a functional mechanism that significantly restricts the range of immediate changes to this massive information store in LTM. The concept of working memory (WM) as a locus of conscious information processing and shortterm maintenance signifies this feature in most cognitive models.

\section{SOURCES OF COGNITIVE LOAD IN VIRTUAL LEARNING ENVIRONMENT}

Virtual reality always provides a metaphorical parallel to our real world. Our model takes advantage of the fundamental role of metaphor to design, structure and build meaningful virtual reality systems. The learning of source knowledge can be made easier by its metaphorical embodiment in artificial worlds, where learners can visualize, experience and interpret this knowledge directly. Even though we borrow most components of our vast knowledge base from other sources rather than discover or build them from the scratch, we always actively reconstruct this knowledge within our WM. The borrowed elements of information are reorganized and integrated with available prior knowledge structures from LTM. In situations where information cannot be borrowed from other sources, 
we use our default problem-solving mechanisms based on search for possible solution steps followed by tests of their effectiveness. Since these processes occur within limited WM, they impose a heavy cognitive load and leave minimal, if any, cognitive resources for meaningful construction of organized knowledge structures in LTM, thus providing inadequate conditions for learning. Some people are more, and some less, influenced by the context when performing a skill or learning[1].

Another part of the essential cognitive load is caused by virtual learning activities and resource that are intentionally designed to enhance acquisition of appropriate schematic knowledge structures. Because these types of cognitive load are essential for comprehending material and constructing new higher-level knowledge, it is important to provide all the necessary resources to accommodate this cognitive load without exceeding WM capacity.

\section{HOW TO REDUCE COGNITIVE LOAD IN VIRTUAL LEARNING ENVIRONMENTS}

There are many factors of influencing cognitive load. But learning tools, learning agents, learning activity and resource architecture play an important role in virtual reality environment. Four factors are depicted in Fig. 1



Figure 1. Influencing Factors of Cognitive Load

\subsection{Design Learning Tools in Virtual Learning Community}

Learning tools are critical to locating, accessing, and manipulating the needed resources, as well as interpreting and evaluating the usefulness of the resources in VLE. Tools enable users to organize and present their understanding in various ways. Searching, processing, manipulation, and communication tools are among those commonly used[2].

Searching tools range from people, such as reference librarians or media specialists, to sophisticated search services that provide specialized search capabilities, to card catalogs providing author, title, and subject searching.

Processing tools provide cognitive support to aid in collecting, organizing, integrating and generating information.

Manipulation tools provide the means to test beliefs, ideas, and theories. Manipulating the features of a roller coaster, learners explore relationships among motion, force, speed, energy, and mass, engaging in thinking as they analyze problems, and then propose and test potential solutions.

Communication tools enable the sharing of ideas in a variety of forms: text, audio, and video. By using asynchronous communication tools such as email and QQ, participants can readily exchange ideas independent of time and place.

\subsection{Design Learning Agents for Virtual Learner Profile}

Establish the student profile: age, level of background knowledge, whether or not they have any physical or cognitive impairment involving special educational needs, their previous experience in VLE, as well as any other characteristic possibly influencing learning.

Resource location and maintenance agents can utilize semantic web metadata to help locate in virtual reality systems and classify new learning materials as well as to monitor existing resources to determine if they have been changed, moved, or eliminated. These agents are similar to information retrieval agents that have been used in other domains[3]. However, resource location agents need to allow semantic searches of online content to facilitate discovery of appropriate materials based on the conceptual meaning of the subject material.

The development of systems utilizing reusable learning objects and the semantic web is for virtual learner profile; however, there are a few implemented systems that show the promise these technologies hold. Utilizing an agent based resource location process can help both instructors and individual learners locate and utilize learning resources that facilitate active learning and knowledge formation (e.g. video, Java applets and Flash materials that provide animated demonstrations and interactivity in VLE. Materials that are deemed appropriate can then be automatically added to lesson plans as needed to support specific learning objectives. 


\subsection{Design Learning Activity as Scaffolding} Strategy in Virtual Learning Group

Scaffolding has been used to facilitate learners in coping with the challenges of complex problems solving in virtual learning group. Scaffolds can be defined as temporary supports provided by the teacher or another student to help learners bridge the gap between their current abilities and the intended goal[4]. The techniques of navigation have to provide the means of selecting the desired direction in VLE; selection of the speed and acceleration of activity; and the means by which learners decide to start, continue and stop activity.

In order to learn, learners need to attend to and process elements of new information, establish key connections between them, integrate them with available knowledge base, and build new or modified knowledge structures. These processes occur in WM and inevitably impose a cognitive load.

According to Vygotsky, learners should be guided or scaffolded by a more capable peer to solve a problems or carry out a task that would be beyond what they could accomplish independently. Scaffolding can help learners accomplish more than what they could accomplish by themselves by providing them with conceptual, metacognitive, procedural, and strategic support that can assist them as they approach and solve ill-structured problems.

Activities have been found to be effective in facilitating problems-solving processes, including problems representation, developing solutions, making justifications, and monitoring and evaluation in VLE. Activity can support learners in representing problems by directing their attention to important characteristics, helping them identify relevant information, eliciting explanation, and activating their prior knowledge. Activity also can support learners as they organize, monitor, and evaluate by making their thinking and learning process explicit.

\subsection{Design Resource Architecture in Virtual Learning Environment}

Users immersed in a VLE get the feeling that they are actually there in the real place. Learners are carried off to an environment of pure information that they can see, listen to and touch. In VLE where presence is high, the sensation of immersion is so strong that the interface disappears and users lose all notion of interacting with a machine. Users can make use of a range of ways of manipulating and modifying virtual worlds. Learners could move the virtual objects by hand, eye movement or voice.

Resource architecture is the science of designing the labeling, navigation, organization and search systems to help people find and manage information more successfully. It is closely aligned with web usability and its importance to large scale website design is increasing as more and more information is stored on servers and a wider range of people use the web. Within the scope of web development, resource architecture involves the logical deconstruction, labeling and categorizing of the mass of information.

Reducing extraneous load caused by resource in VLE. The effects could be moderated by the levels of learner expertise. For learners with lower levels of prior knowledge, pacing may not be effective on its own and need to be supplemented with segmenting materials into smaller sections. Methods for representation control may help to balance the amount of information and reduce spatial split attention by selecting appropriate modes of presentations, and to direct learner attention by displaying appropriate visual cues. Content control methods could also balance the amount of information provided to individual learners according to their needs and available task-specific knowledge base. For example, more experienced learners could themselves segment the content and appropriately sequence the segments, thus facilitating knowledge construction. Content control methods could often be effective only for relatively more experienced learners who have sufficient prior knowledge of the task domain. To reduce the influence of missing prior knowledge on the effectiveness of interactive learner-controlled environments, we suggested including pre-training sessions that would activate or provide learners with relevant prior knowledge (the pre-training principle).

\section{APPLY METAPHORICAL PROJECTION TO VIRTUAL LEARNING ENVIRONMENT}

One of the key questions in VLE is to properly select the source knowledge. Obviously, it makes no sense to teach all educational contents using this technology, either because they can be directly learnt using traditional techniques or because other educational technologies are more effective and cheaper. Implement a preliminary design of the virtual world by means of a metaphorical projection, creating a meaningful isomorphism between the knowledge of the real environment and the virtual world for learners. This preliminary design will serve to establish how the didactic material should be presented and structured, as well as to define the role of learners and teachers in the virtual world.

For example, the goal of the first task-learning of the taxonomic conceptual domain is to distinguish the main key concepts of any classification: hierarchy, class, category, properties, inheritance, classificational criteria, etc. Learners should be able to discover the metaphorical meaning of the virtual world by exploring the scenario, interpreting each of its elements and correctly relating these elements to the key concepts. 
The main features offered in VLE are as follows:

- Presence: users immersed in VLE get the feeling that they are actually there in the real place. Learners are carried off to an environment of pure information that they can see, listen to and touch. In artificial worlds where presence is high, the sensation of immersion is so strong that the interface disappears and users lose all notion of interacting with a machine.

- Navigation: learners can be either immobile observers or travelers in VLE, moving around in different ways, e.g., walking, using a vehicle, touching an object or pointing in any direction within the environment, etc.

- Viewpoint: this is the possibility of users changing perspective at will. For example, learners could pass on their viewpoint to a given artificial world object or process, or even to the viewpoint of another participant. There are two possible user viewpoints: one is egocentric, when users are within the scenario, inside a room-cube. The other is exocentric, if users have the possibility of exiting, moving outside of the scenario and looking at the cube network from a distance. Thus, the full hierarchy could be visualized as if it were a 3D map in VLE.

- User-environment interaction: users can make use of a range of ways of manipulating and modifying virtual worlds. Learners could move the virtual objects by hand, eye movement.

The interaction with objects encompasses two basic tasks in VLE: the selection of the objects and how they can be manipulated. With regard to the interaction with objects for our scenario, the best thing would be direct manipulation. However, there are other options: move objects with eyes, gestures, verbal orders or menus. Where pedagogically necessary or relevant, some objects could be permitted to have magical features or learners could be given the possibility of directly altering the environment.
Co-operative learning: distributed and networked environments provide for collective participation, offering several users the possibility of sharing virtual spaces at the same time. Accordingly, the real-time interaction between different learners leads to genuine co-operative learning.

\section{CONCLUSION}

Visualization of information and sources are located in VLE. It can be used to understand and solve scientific problems, look for regularities or connections. Most of the approaches and techniques used may generate both essential and unnecessary cognitive processes, and contribute to both reduction and increase in cognitive load. Learner cognitive characteristics should be taken into account rather than relying rigidly on some fixed principles. The aim is to transfer learner behavioral activity into cognitive interactivity that generates essential cognitive processing relevant to knowledge construction without increasing non-essential extraneous processing load in VLE.

\section{REFERENCES}

[1] Jianzhen Chen, A Cognitive Approach to Design Case for e-Learning, GCSE2011

[2] Rezazadeh, I. M., M. Firoozabadi, M., \& Wang, X. Evaluating the usability of virtual environment by employing affective measures. Intelligent Systems, Control and Automation: Science and Engineering, 1010, 95109,2011

[3] Wouters, P., Tabbers, H. K., \& Paas, F. Interactivity in video-based models. Educational Psychology Review, 19. DOI 10.1007/s10648-007-9045-4, 2007.

[4] S. M. Huang, H.Y. Hsueh, J.S. Hua, "Discovery of educational objective on E-Learning resource: A competency approach", 6th International Conference on Web Based Learning, 2007 